\title{
Analysis of expansion within a pressure inflated section of an urethral stricture model.
}

\begin{abstract}
The Urethra is a long tubular structure in the genitourinary tract and serves important functions. Researchers have experimented with some approaches to model the urethra and to analyse its biomechanical properties. However, experiments to model the in-vivo behaviour of urethra with strictures is not thoroughly explored. To analyse the in-vivo urethral properties and specifically for supporting treatment of strictures, a new inflatable sensor-actuator system is being developed. The capabilities of this sensor shall be evaluated in simulations which require appropriate modelling of the human male urethra with strictures. This forms a part of the identification procedure for a variety of urethra conditions and geometries, which in turn forms a basis for inverse modelling. As an initial simplified approach, an axisymmetric Finite Element model was generated that resembled the urethra incorporating a stricture region. An ideal actuator with sensor elements exerting a pressure on inner wall of this urethra was simulated. Three circumference measurement zones within the sensor height (top surface, centre and bottom surface) were implemented. The resulting pressure-extension (circumferential) responses were determined at these measurement zones. The sensor was placed at different lengths within this urethral tube and inflated and the pressure-extension responses were noted. It was found that depending on the position of the sensor-actuator, the extension of tissue can vary. The possible factors for this variation were the finite length of the actuator as well as the influence of tissue properties around the measurement zones. This is important information for the interpretation of sensor data to be gained by the current development. It was possible to generate datasets based on an ideal sensor model, that proved helpful in the evaluation of biomechanical tissue properties in healthy and stricture conditions. This indicates simulations are a versatile and prospective way to test new sensors prior to real experiments.
\end{abstract}

Keywords: modelling, urethra, strictures, simulation.

Corresponding author: Ashish Bhave: Institute of Technical Medicine, Furtwangen University, Jakob-Kienzle-Straße 17, Villingen-Schwenningen, Germany, e-mail: bha@hsfurtwangen.de

Knut Möller: (moe@hs-furtwangen.de): Institute of Technical Medicine, Furtwangen University, Villingen-Schwenningen, Germany https://doi.org/10.1515/cdbme-2021-2147

\section{Introduction}

The Urethra is a long tubular structure in the human genitourinary tract that allows passage of urine and other fluids. Strictures are a pathological change that consists of tissue hardening and/or constriction within the urethra. They may occur after injury to the epithelium of the urethral lumen and/or underlying corpus spongiosum. This can cause hardening of tissue due to body repair mechanisms. A minimally invasive procedure for dilatation of the urethra (at stricture site) is proven to provide relief to the patient, but the recurrence of the problem is found in up to $85 \%$ of investigated cases $[1,2]$

Urologists find it challenging to determine the appropriate pressure/force to open the constricted vessel (provided it also prevents the urethra from undergoing plasticity or rupture). By processing active data from an intraluminal sensor, with relevant techniques, it can ultimately provide necessary information that supports decision making for the Urologist(s). At two research institutes of Furtwangen University, the project ILSA (Intra-Luminal Sensor Actuator) is being undertaken. An intra-luminal sensor is currently being developed that may provide pressure-extension features of the vessel in-vivo during a clinical procedure [3].

Some authors have undertaken mathematical modelling of the urethra [4,5]. The current literature lacks comparable studies, and it was found that the urethra is a biomechanically not well investigated organ. Urethral Finite Element Method (FEM) models are based on physics and causality and are thus able to predict a tissue response to a stimulus ("Forward model"). The identification of the actual urethra tissue condition from sensor data is called reverse or inverse modelling. This paper aims at exploring the different aspects of forward modelling and whether simulated sensor readings (an identification dataset) are sufficiently informative to reconstruct the tissue parameters from the model. The aim is to generate urethral stricture models and simulate expansion of different areas within this tube. Using an FEM model, it is intended to analyse these pressure-extension responses which may aid the modelling process. 


\section{Methods}

In the subsequent sections, we propose a simulation model to generate circumference over pressure curves for different measurement planes.

COMSOL Multiphysics (v5.5) non-linear and structural module add-ons were used for the simulations [6]. Gasser et al., described a constitutive model for the arteries considering collagen fibre orientation and its distribution. This equation is a stable modification derived from the classical Holzapfel Gasser Ogden (HGO) model for arteries [7,8]. An anisotropic hyperelastic response could be generated in their implementations [8]. The strain energy density equation defined by Gasser et al., was chosen as the mathematical equation for the modelling. It is defined as follows:

$$
\begin{gathered}
\mathrm{W}=\mu\left(I_{1}-3\right)+ \\
\frac{k_{1}}{2 k_{2}} \sum_{\alpha}\left(e^{k_{2}\left[\kappa\left(I_{1}\right)+(1-3 * \kappa)\left(I_{\text {fib }}^{\alpha}\right)-1\right]^{2}}-1\right)
\end{gathered}
$$

$\mathrm{W}$ is the strain energy density. $\mu$ is the isotropic part constant of the neo-hookean material. $I_{1}$ is the first invariant of the tensor. It is a Cauchy normal stresses trace on the tensor. $\kappa$ is a structural constant. The fibre network consists of two families of fibres with material properties $k_{1}$ and $k_{2} . k_{1}$ is the stress parameter expressed in pressure while $k_{2}$ is a dimensionless parameter. $\left(I_{\mathrm{fib}}^{\alpha}\right)$ is the invariant which describes the stretch of the fibre family in consideration. The fibres contributed to strain energy density only when there is no buckling i.e. the contribution occurs only when $I_{f i b}^{\alpha}$ was greater than 1 .

Table 1: Gasser equation parameters for 'Healthy' and 'Fibrosis' tissue regions.

\begin{tabular}{|l|l|l|l|l|l|l|}
\hline & $\begin{array}{l}\boldsymbol{\mu} \\
\mathbf{( k P a )}\end{array}$ & $\begin{array}{l}\boldsymbol{k}_{\mathbf{1}} \\
\mathbf{( k P a )}\end{array}$ & $\boldsymbol{k}_{\mathbf{2}}$ & $\boldsymbol{\kappa}$ & $\begin{array}{l}\text { Fibre } \\
\mathbf{f a m i l y} \\
\mathbf{1}\left({ }^{\circ}\right)\end{array}$ & $\begin{array}{l}\text { Fibre } \\
\mathbf{f a m i l y} \\
\mathbf{2}\left({ }^{\circ}\right)\end{array}$ \\
\hline Healthy & 5 & 2.5 & 1 & 0.2 & $40^{\circ}$ & $140^{\circ}$ \\
\hline Fibrosis & 60 & 200 & 100 & 0.2 & $40^{\circ}$ & $140^{\circ}$ \\
\hline
\end{tabular}

The cylindrical tube model representing a section of the urethra is modelled and described in subsequent parts. The cylinder has 2 fibre families for any region. ' $\alpha$ ' $=1,2$ depict the two fibre families. Incompressibility condition was applied to the entire tissue model. The model described by Gasser et al., is shown to adhere to the polyconvexity and stability conditions. The parameters for the gasser equation for the 'healthy' and 'fibrosis'(hardened/stricture) tissue are provided in Table 1.

An axisymmetric model mode in COMSOL is used to create a hypothesized section of an urethra. Thus, a cylinder with an inner radius of $4 \mathrm{~mm}$ and outer radius of $9 \mathrm{~mm}$ was created. The thickness of the urethra, was chosen to be $5 \mathrm{~mm}$. The cylinder height was chosen as $60 \mathrm{~mm}$. In the axisymmetric modelling setup, the cylinder was observed constructed from a rectangle that was extruded $360^{\circ}$ along the axis ' $r=0$ '. The base and the top of the cylinder was given a symmetric

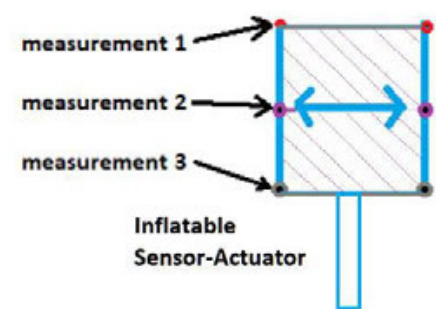

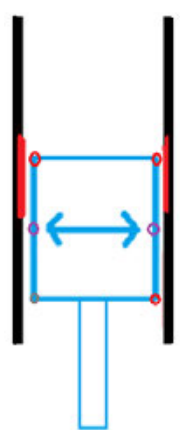

(b) (a)

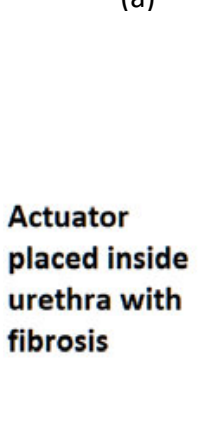

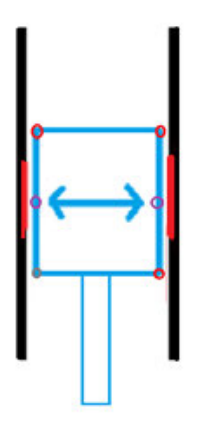

(c)
Figure 1: (a)-Measurement locations(planes) of the inflatable sensor-actuator (b) Cross-section view of actuator placement just inside the urethra cylinder-'simulation 1'. The urethral wall is the black coloured segment and contains 'red' colour fibrosis region within the segment. (c) Sensor placement deeper inside the urethra -'simulation 2'. The urethral wall is the black coloured segment and embeds 'red' colour fibrosis region within the segment.

constraint. The meshing was performed for the rectangle that contained 218 fine rectangular mesh surfaces and 259 nodes points. In the central portion of the cylinder that measured $10 \mathrm{~mm}$ - (the section in the height portion from $25 \mathrm{~mm}$ to $35 \mathrm{~mm}$ ), consists of fibrosis (hardened area as 'red' region in Figure $1(b, c))$. The other portion is the healthy tissue.

The sensor provided circumference measurements at three 'measurement locations' over the ideal sensor-actuator setup (see Figure 1(a)). The measurements are induced by inflation of the actuator (balloon) and measuring the resultant circumferential expansion. This sensor-actuator system is a cylinder of $2 \mathrm{~cm}$ height and $4 \mathrm{~cm}$ radius. This sensor is placed inside the tube that contains a 'stricture' zone (red coloured region embedded in the black tube section) (see Figure1(b,c)). In 'simulation 1', the actuator is placed so that it leaves $10 \mathrm{~mm}$ 
height from the bottom of the tube. The measurements from the three 'planes' are obtained. In a similar manner, the measurements in 'simulation 2' are obtained when the actuator left $20 \mathrm{~mm}$ height space from bottom of the tube. Both simulations provide measurements taken from 'fibrosis' zone and form the 'healthy' tissue. A comparison of the resulting pressure-extension slopes and values within these three measurement locations was performed for each simulation set. A comparison of the pressure-extension outputs was also performed based on the material property of urethra ('healthy' and 'fibrosis') in these two simulations.

\section{Results}

The pressure-extension response of a urethral wall segment was obtained (see Figure 2). The uninflated circumference for all the models was found to be $\sim 25 \mathrm{~mm}$ which matched the original inner circumference of the tube. It was observed that as the input pressure increases, the simulated sensor extension values increase albeit with a saturation (see Figure 2 and 3).

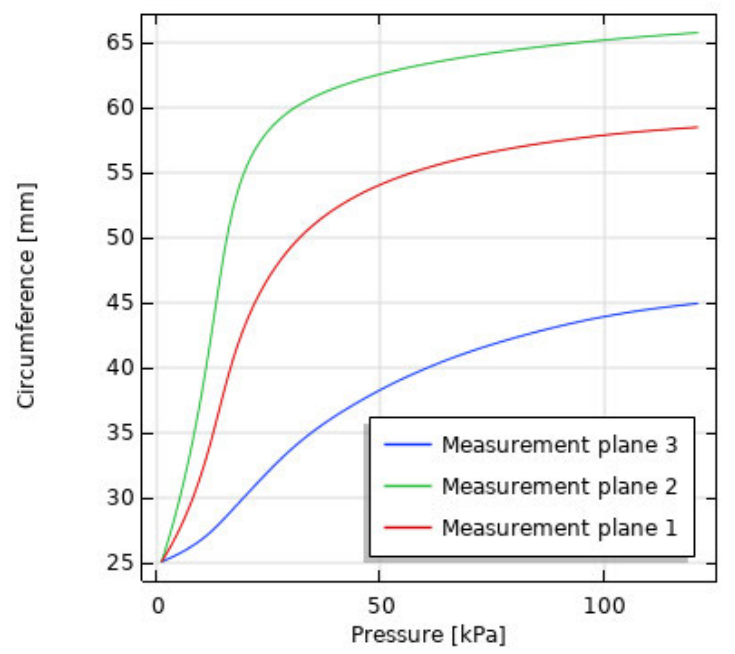

Figure 2: Simulation 1 showing pressure-circumference response within the sensor. The healthy tissue at the central portion of the sensor extends the most (plane 2). The healthy tissue at the top of the sensor (plane 1) extends less in comparison, while the tissue at the fibrosis region extends the least.

When the sensor is inflated in simulation 1 , at $120 \mathrm{kPa}$ input pressure, at measurement planes ' 1 ', '2' and ' 3 ', a circumferential extension of $58 \mathrm{~mm}, 66 \mathrm{~mm}$ and $45 \mathrm{~mm}$ was found, respectively. The central portion of the ballon-actuator extends the most (plane 2-lies at healthy tissue area) (see Figure 2). The sensor expansion at the healthy tissue in 'measurement plane 1' extends less in comparison to the central portion at similar pressures. The plane that lies at the fibrosis tissue (measurement plane 3) shows the least extension among the three, throughout the pressure range.

When the sensor is inflated in simulation 2 , at $120 \mathrm{kPa}$ input pressure at measurement planes ' 1 ', '2' and ' 3 ', a circumferential extension of $61 \mathrm{~mm}, 50 \mathrm{~mm}$ and $61 \mathrm{~mm}$ was found respectively. The sensor expansion at the planes 1 and 2 (at healthy tissue) was identical and their response overlap each other. The central portion of sensor (at the fibrosis tissue) response is stiffer in comparison throughout the pressure range (see Figure 3).

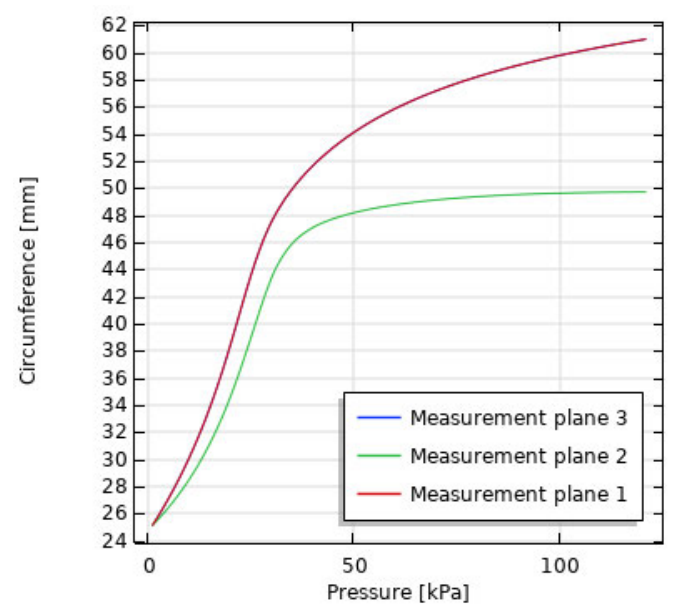

Figure 3: Simulation 2 showing pressure-circumference response within the sensor. The healthy tissue at the top and bottom of the sensor (plane 1 and 3 ) have identical response and extend more in comparison with the tissue at the fibrosis region at the centre of sensor region (plane 2).

'Measurement Plane 3' in simulation 1 and 'measurement plane 2' in simulation 3 lie on the fibrosis (hardened) region. At pressure of $120 \mathrm{kPa}$, circumference extension of $45 \mathrm{~mm}$ and $50 \mathrm{~mm}$ was obtained for simulation 1 and 2, respectively. For simulation 1 , the response was stiffer at lower pressures when compared with simulation 2 . For healthy tissue portion within the tube, at pressure of $120 \mathrm{kPa}$, variation of circumference extension values ranging $58 \mathrm{~mm}$ to $66 \mathrm{~mm}$ were obtained for both the simulations.

\section{Discussion}

All the pressure-extension curves of the graph show a general response of a hyperelastic material. Beyond certain pressures (for each curve different) the slope of circumferential extension tapers (still being positive). The model shows a capability to tweak the response by changing the values of the parameters of the chosen mathematical model. Extensive studies using the 'gasser' equation have been performed in the 
arterial domain and also to some extent in other biological tissues. The range of values for the parameters for this equation is extensive and varies depending on tissue, nature of experiments as well as approximations. However, literature lacks relevant model parameter values for the urethra and its stricture. Though within range found for biological elastic tissues, the values of the model parameters in this study are not based on histology or validated experiments and are thus experimental in nature. The pressure range was chosen based on limits of urethral rupture and pressures the urologists may use for inflatable balloon catheters during treatment of strictures in a clinical setup [9].

At pressure of $120 \mathrm{kPa}$, circumference extension of $45 \mathrm{~mm}$ and $50 \mathrm{~mm}$ was obtained for simulation 1 and 2, respectively. For simulation 1, the response was stiffer at lower pressures when compared to simulation 2. The reason for stiffness in simulation 1 may be because only a portion within the stricture portion was inflated leaving the other part out. The uninflated fibrosis tissue exerts a pulling force that dampens the inflation at this section. In contrast, for simulation 2 , the entire stricture portion is inflated. This is indicative that expansion of the urethral tube may rely not only on the property of the tissue, but also the section where the sensor is inflated. The finite length of the sensor would likely have an impact additionally.

For healthy tissue portions within the tube, at pressure of $120 \mathrm{kPa}$, a variation of circumference values varying from $58 \mathrm{~mm}$ to $66 \mathrm{~mm}$ was obtained. If the geometry of the tube were tweaked, the response could have been different. The presence of hardened tissue may have an impact on how the pressure redistributes itself within the sensor. This might be the reason for obtaining a non-uniform response for tissues with similar initial circumference and other properties.

The material description chosen used a mathematical model based on the fibre stretch. In simulation, while the tube expands, there may be sections where the pressure leads to buckling of the material at certain non-pressure portions. It is possible that implementing different boundary conditions may provide varied results.

A general investigation of how an inflatable sensor would expand in different sections of an urethra tube was undertaken. It was observed from both experiments, that a variation in the extension characteristics at fibrotic tissue and healthy tissue exists and is influenced by the placement of the sensoractuator within the tube

\section{Conclusion}

Approaches to model the behaviour of urethral wall tissue and the process of forward and reverse modelling is being undertaken. The outputs from simulations are indicative that the expansion of the urethral tube relies not only on the property of the tissue, but also the section where the sensor is inflated. Multiple test scenarios could be conceptualized to mimic in-vivo situations. Verification and validation of these outputs with in-vivo experiments would be the next step towards refining the models.

\section{Author Statement}

This research was partly supported by the German Federal Ministry of Research and Education(BMBF) under grant no. 13FH5I05IA (CoHMed/Digitalization in the OR).

\section{References}

[1] Hampson LA, McAninch JW, Breyer BN. Male urethral strictures and their management. Nature reviews. Urology 2014; 11: 43-50.

[2] E. Martins F, Simoes de Oliveira P, M. Martins N. Historical Perspective and Innovations in Penile Urethroplasty. In: Pang R, editor. Lower Urinary Tract Dysfunction - From Evidence to Clinical Practice. London: IntechOpen; 2020.

[3] Sittkus B. ILSA-IP05 project meet. Sensor-Actuator development and progress January 2021.

[4] Masri C, Chagnon G, Favier D, Sartelet H, Girard E. Experimental characterization and constitutive modeling of the biomechanical behavior of male human urethral tissues validated by histological observations. Biomechanics and modeling in mechanobiology 2018; 17: 939-950.

[5] Cunnane EM, Davis NF, Cunnane CV, et al. Mechanical, compositional and morphological characterisation of the human male urethra for the development of a biomimetic tissue engineered urethral scaffold. Biomaterials 2021; 269: 120651.

[6] COMSOL. COMSOL Multiphysics Reference Manual: https://doc.comsol.com/5.5/doc/com.comsol.help.comsol/CO MSOL_ReferenceManual.pdf; 2019.

[7] Holzapfel GA, Gasser TC, Ogden RW. A New Constitutive Framework for Arterial Wall Mechanics and a Comparative Study of Material Models. J Elast 2000; 61: 1-48.

[8] Gasser TC, Ogden RW, Holzapfel GA. Hyperelastic modelling of arterial layers with distributed collagen fibre orientations. Journal of the Royal Society, Interface 2006; 3 : 15-35.

[9] Davis NF, Cunnane EM, Mooney RO', Hess J, Walsh MT. Characterisation of human urethral rupture thresholds for urinary catheter inflation related injuries. Journal of the mechanical behavior of biomedical materials 2018; 83: 102 107. 